\title{
THE HOMOTOPY TYPE OF TWO-REGULAR $K$-THEORY
}

\author{
Luke Hodgkin and Paul Arne Østvær
}

\begin{abstract}
We identify the 2-adic homotopy type of the algebraic K-theory space for rings of integers in two-regular exceptional number fields. The answer is given in terms of well-known spaces considered in topological K-theory.
\end{abstract}

\section{Introduction.}

Let $E$ be a number field, $\mathcal{O}_{E}$ its ring of algebraic integers, and $R_{E}=\mathcal{O}_{E}\left[\frac{1}{2}\right]$ the corresponding ring of 2-integers. For the definition of the algebraic K-theory space $K\left(\mathcal{O}_{E}\right)$ (resp. $K\left(R_{E}\right)$ ) we refer to [Q1]; it is the usual 'plus construction' on the stable classifying space $B G L\left(\mathcal{O}_{E}\right)$ (resp. $B G L\left(R_{E}\right)$ ). A related, homotopically more accessible space is the étale topological K-theory space $K\left(R_{E}\right)$ ét of $R_{E}$, see [D-F1]. The purpose of this paper is to pin down the 2-adic homotopy type of $K\left(R_{E}\right)$ for some special $E$. Our results rely on the following recent advances.

In [R-W1], Rognes and Weibel determined up to extensions the 2-completed groups $K_{n}\left(R_{E}\right) \hat{2} \quad\left(=K_{n}\left(\mathcal{O}_{E}\right) \hat{2}\right.$ for $\left.n \geq 2\right)$. Their computation is expressed in terms of the étale cohomology groups $H^{*}\left(R_{E} ; \mathbb{Z}_{2}(i)\right)$ of $R_{E}$ with coefficients twisted by the action of the roots of unity. A case where the extension problems disappear is the '2-regular case', (see definition below) for which Rognes and Østvær [R-Ø] gave a complete description of $K_{n}\left(R_{E}\right) \hat{2}$, for all $n$. The above results are among the consequences of Voevodsky's solution of the Milnor conjecture [V], as developed in subsequent work [S-V], [B-L]. A particular interesting end-product is 'étale descent for K-theory of number fields at 2', i.e. the strong Quillen-Lichtenbaum conjecture is true for number fields at the prime 2. See [R-W2] for the case of totally imaginary number fields, and [Ø1] for the case of real number fields. In particular, we have:

(a) the spaces $K\left(R_{E}\right)$ and $K\left(R_{E}\right)$ ét are the same on zero-connected components at the prime 2 , and

(b) in consequence one can try to adopt the étale homotopy methods from [D-F1] to determine the 2-completed homotopy type of $K\left(R_{E}\right)\left(=K\left(\mathcal{O}_{E}\right)\right.$ on one-connected components).

Part (b) was solved in the case where $E$ is 2-regular and 'non-exceptional' (see definition below), in [Ø2]; the result is satisfyingly simple, in that the homotopy type is a product of well-known components. In this paper we apply a similar analysis to the more complicated 'exceptional' case. Here the product structure is replaced by a twisting (fibration), but the degree of complication is minimal, and

1991 Mathematics Subject Classification. 19D99, 55N15, 55P15.

Key words and phrases. Homotopy type of K-theory spaces, rings of integers in number fields. The second author was supported in part by NSF. 
almost all of the factors are untwisted, and again of a simple type. As a check, we verify that our result gives the homotopy groups calculated in $[\mathrm{R}-\varnothing]$.

To state the results, we need some definitions and notation. Let $r_{1}$ respectively $r_{2}$ denote the number of real embeddings respectively pairs of conjugate complex embeddings of $E$. We say that $E$ is 2-regular if any of the following equivalent conditions is satisfied:

1) The 'modified tame kernel' of $E$ vanishes. This latter can be identified with the kernel of the natural surjection:

$$
\alpha^{2}: H^{2}\left(R_{E} ; \mathbb{Z}_{2}(2)\right) \rightarrow \bigoplus^{r_{1}} H^{2}\left(\mathbb{R} ; \mathbb{Z}_{2}(2)\right) \cong r_{1} \cdot \mathbb{Z} / 2
$$

summed over the real embeddings of $E$.

2) The ideal (2) does not split in $E$ and the narrow Picard group Pic $+\left(R_{E}\right)$ of $R_{E}$ has odd order.

3)

$$
H^{2}\left(R_{E} ; \mathbb{Z}_{2}(i)\right)=\left\{\begin{aligned}
r_{1} \cdot \mathbb{Z} / 2 & (i \neq 0 \text { even }) \\
0 & (i \neq 1 \text { odd })
\end{aligned}\right.
$$

For a discussion, and further criteria, see proposition 2.2 of [R- $\varnothing]$.

Next, let $\zeta_{m}$ denote an $m$ th root of unity and $\bar{\zeta}_{m}$ its conjugate. For each $r$, we have the cyclotomic extension $E\left(\zeta_{2^{r}}\right) . E$ is said to be exceptional if $\operatorname{Gal}\left(E\left(\zeta_{2^{r}}\right) / E\right)$ is not cyclic for some $r$; otherwise it is non-exceptional. It is easy to check that $E$ is non-exceptional if and only if $\zeta_{4} \in E$ or $\zeta_{2^{k}}+\bar{\zeta}_{2^{k}} \in E$ for some $k \geq 3$. Any real number field is exceptional, while any 2-cyclotomic field $\mathbb{Q}\left(\zeta_{2^{r}}\right), r \geq 2$, is nonexceptional. In our case (exceptional), the description of $K\left(\mathcal{O}_{E}\right)_{2} \hat{~ i s ~ c o m p l i c a t e d ~}$ by the above-mentioned twisting, and we require some names for the spaces which will be our building blocks.

We consider all spaces completed at the prime 2, where not explicitly stated; we hope that the reader will accept statements such as ' $\pi_{1}\left(S^{1}\right)=\mathbb{Z}_{2}$ ' which result from this. We also abusively write $\operatorname{Spec} R$ for what is properly the étale homotopy type $(\operatorname{Spec} R)_{\text {ét }}$. As usual $U, O$ are the stable unitary and orthogonal groups, and $B U, B O$ their classifying spaces. The complexification $c$ maps $O$ into $U$ as a subgroup, with quotient $U / O$. For $q$ odd, let $\psi^{q}$ be the Adams operation on $B U$. Note in particular that $\psi^{-1}$ is the conjugation map. By Quillen's fundamental result [Q2], when $q$ is an odd prime-power the $K$-theory space of the finite field $\mathbb{F}_{q}$ is the fibre of $\psi^{q}-1: B U \rightarrow B U$; we shall denote this space by $F \psi^{q}$. Similarly, we write $F \psi^{-1}$ for the fibre of $\psi^{-1}-1$. We require two variants of $F \psi^{q}$ :

1) In analogy with a construction of Bökstedt, define $J K(q)$ to be the fibre of the composite:

$$
B O \stackrel{c}{\rightarrow} B U \stackrel{\psi^{q}-1}{\longrightarrow} B U
$$

(This is $K(\mathbb{Z})$ if $q \equiv \pm 3 \bmod 8$.)

2) Let $j: F \psi^{-1} \rightarrow B U$ be the inclusion of the fibre, and define $J_{c}(q)$ to be the fibre of the composite $\left(\psi^{q}-1\right) \circ j: F \psi^{-1} \rightarrow B U$. (Note the analogy between this construction and the previous one.)

Our next points concern Galois groups. Let $\mu_{\infty}(E)$ respectively $\mu_{\infty}$ be the group of 2-primary roots of unity in $E$ respectively $\mathbb{C}$, and let $\Gamma_{E}^{\prime}=\operatorname{Gal}\left(E\left(\mu_{\infty}\right) / E\right)$. The natural action of $\Gamma_{E}^{\prime}$ on $\mu_{\infty}$ gives a monomorphism 
- compare $\S 1$ of [Mi2]. By considering $\pi_{1}\left(\operatorname{Spec} R_{E}\right)$ as the Galois group of the maximal unramified extension of $R_{E}$, we see that the action of $\pi_{1}$ (Spec $R_{E}$ ) on $\mu_{\infty}$ factors through $\Gamma_{E}^{\prime}$ to give a composite:

$$
\pi_{1}\left(\operatorname{Spec} R_{E}\right) \rightarrow \Gamma_{E}^{\prime} \stackrel{\phi}{\rightarrow} A u t\left(\mu_{\infty}\right)
$$

which we shall call $\hat{\phi}$. Clearly the images of $\phi$ and $\hat{\phi}$ in $\operatorname{Aut}\left(\mu_{\infty}\right)$ are the same subgroup, say $\Lambda$. If $E_{0}=E(\sqrt{-1})$, it is a consequence of the 'exceptional' condition on $E$ that $\Gamma_{E}^{\prime}=\Gamma_{E} \times \mathbb{Z} / 2$, where $\Gamma_{E}=\operatorname{Gal}\left(E\left(\mu_{\infty}\right) / E_{0}\right)$. Still following [Mi2], set $a_{E}=\nu_{2}\left(\left|\mu_{\infty}\left(E_{0}\right)\right|\right)$ (the 2-adic valuation). Then $\Lambda$ is (topologically) generated by

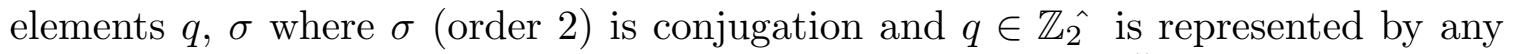
integer such that $q$ is $\equiv \pm 1 \bmod 2^{a_{E}}$ but not $\bmod 2^{a_{E}+1}$. By Čebotarev's theorem we can always choose a prime $\mathfrak{P}$ in $R_{E}$ such that the order $q$ of the finite field $R_{E} /(\mathfrak{P})$ is an integer with these properties.

For future reference, we define numbers $w_{m}=w_{m}(E)$ by: $w_{m}=2$ ( $m$ odd), $w_{m}=2^{a_{E}+\nu_{2}(m)}(m$ even). (Compare e.g. the definition in [R-W1], which is equivalent in the exceptional case. Mitchell [Mi2] writes $w_{i}$ for the exponents, rather than the powers of 2.)

Our main result is as follows:

Theorem 1.1. With the preceding notation, let $E$ be 2-regular and exceptional. Then at the prime 2:

(i) If $E$ is totally imaginary $\left(r_{1}=0\right), K\left(R_{E}\right)$ is homotopy equivalent to the product

$$
J_{c}(q) \times \prod^{r_{2}-1} U
$$

( $r_{2}-1$ factors in the product)

(ii) If $r_{1}>0, K\left(R_{E}\right)$ is homotopy equivalent to the product

$$
J K(q) \times \prod^{r_{2}} U \times \prod^{r_{1}-1}(U / O)
$$

Our strategy in proving this result is based on ideas present in [D-F1], and is as follows. We find a space $X$, and map $f: X \rightarrow \operatorname{Spec} R_{E}$ which induces an isomorphism on mod 2 homology. The space $X$ is a wedge of $r_{2}+1$ circles and $r_{1}$ copies of the infinite projective space $R P^{\infty}$. The key question is then how the wedge components of $X$ map via the composite:

$$
\pi_{1}(X) \stackrel{f_{*}}{\rightarrow} \pi_{1}\left(\operatorname{Spec} R_{E}\right) \stackrel{\hat{\phi}}{\rightarrow} \operatorname{Aut}\left(\mu_{\infty}\right)
$$

Specifically, the condition required is that $\operatorname{Im}\left(\hat{\phi} \circ f_{*}\right)=\operatorname{Im}(\hat{\phi})=\Lambda$. According to [D-F1], in the given circumstances, $K\left(R_{E}\right)$ is homotopy equivalent to a space called $K(X)$; and to find $K(X)$ we need to know the wedge components, and the way that their fundamental groups map into $A u t\left(\mu_{\infty}\right)$. We therefore find these, simplify as much as possible, and our theorem will follow from Dwyer and Friedlander's result. As can be seen from the statement of the theorem, cases (i) and (ii) need separate 
It should be noted that all of these computations could have been done at the time of the original paper [D-F1], modulo replacing the $K$-theory spaces by their étale topological versions; indeed, our reliance on the methods of Dwyer and Friedlander is substantial. However, the étale descent results we mentioned in the beginning make it possible to state the results for the $K$-theory spaces themselves.

A general solution to the problem of finding $K$-theory spectra of number rings at the prime 2 has been undertaken by Mitchell [Mi1, Mi2]. The results are fuller, and give in fact the 2-adic homotopy type when combined with [R-W2] and [Ø1]. But their interpretation requires knowledge of the 'Iwasawa module', which appears difficult in general.

\section{The totally imaginary case.}

In this section, $E$ will denote a number field which is 2-regular, exceptional, and totally imaginary. We can deduce immediately (cf. [R-Ø]):

Lemma 2.1. The mod 2 homology of $R_{E}$ is given by:

$$
\begin{aligned}
& H_{1}\left(R_{E} ; \mathbb{Z} / 2\right)=\left(r_{2}+1\right) . \mathbb{Z} / 2 \\
& H_{i}\left(R_{E} ; \mathbb{Z} / 2\right)=0 \quad(i>1) .
\end{aligned}
$$

However, in this case we can do better, since the 2-completed homology is also simple; for this we write $H_{i}(\quad)$, omitting the coefficients.

Lemma 2.2. The 2-completed homology of $R_{E}$ is given by:

$$
\begin{aligned}
H_{1}\left(R_{E}\right) & =\left(r_{2}+1\right) \cdot \mathbb{Z}_{2} \\
H_{i}\left(R_{E}\right) & =0 \quad(i>1) .
\end{aligned}
$$

Proof. Let $\alpha$ be the unique prime above 2 in $\mathcal{O}_{E}$. Then by theorem 2.2 of [D-F1], $H_{1}\left(R_{E}\right)$ is a quotient of $\left(\mathcal{O}_{E}\right)_{\alpha \wedge}^{* \wedge}$ (since the narrow Picard group vanishes); that is, of a direct sum of copies of $\mathbb{Z}_{2}^{\hat{}}$ and 2-torsion groups. From the description of the mod 2 homology in lemma 2.1, there is no 2-torsion, so $H_{1}$ is a sum of copies of

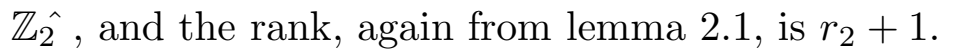

Next, recall from $\S 1$ the homomorphism $\hat{\phi}: \pi_{1}\left(\operatorname{Spec} R_{E}\right) \rightarrow \operatorname{Aut}\left(\mu_{\infty}\right)$. In [Ø2] (following a model from [D-F1]) it was shown that in the non-exceptional case there is a map, $f: X \rightarrow \operatorname{Spec} R_{E}$, inducing an isomorphism on mod 2 homology, where:

(i) $X$ is a wedge of $r_{2}+1$ circles;

(ii) The circles can be chosen in such a way that the first one maps to a topological generator of $\mathbb{Z}_{2}$, and the rest map trivially.

Our first result in this section is parallel to this, if slightly more complicated. It states:

Proposition 2.1. In the exceptional totally imaginary case, there is a map $f$ : $X \rightarrow \operatorname{Spec} R_{E}$ such that:

(i) $X$ is a wedge of $r_{2}+1$ circles;

(ii) The first circle, considered as an element of $\pi_{1}(X)$, maps under $\hat{\phi} \circ f_{*}$ to $q \in \Lambda$;

the second to the non-trivial element $\sigma$ of order 2 in $\Lambda$; and the rest (if any) map 
(iii) $f$ induces an isomorphism on mod 2 homology.

Proof. This is essentially elementary topology, using what we know of the homology of Spec $R_{E}$. In fact, we can clearly choose $r_{2}+1$ maps from $S^{1}$ to $\operatorname{Spec} R_{E}$ whose images under the Hurewicz map generate $H_{1}\left(R_{E}\right)\left(\mathbb{Z}_{2}\right.$ or $\mathbb{Z} / 2$ coefficients). The key adjustments to be made concern their images under $\phi$. We know the structure of $\operatorname{Im}(\hat{\phi})=\Lambda$, and since the latter is Abelian, $\hat{\phi}$ factors through $H_{1}\left(R_{E}\right)$. Hence we can find maps $f_{1}, f_{2}: S^{1} \rightarrow$ Spec $R_{E}$ which represent generators of $H_{1}$, such that $f_{1}$ maps under $\phi$ to $q$, and $f_{2}$ maps to $\sigma$.

Now let $f_{3}, \ldots, f_{r_{2}+1}: S^{1} \rightarrow$ Spec $R_{E}$ represent the remaining generators of $H_{1}$. We can multiply (in $\pi_{1}$ ) by suitable powers of $f_{1}, f_{2}$ to obtain maps $g_{3}, \ldots, g_{r_{2}+1}$ which still define a basis of $H_{1}$ together with $f_{1}, f_{2}$, and map trivially under $\hat{\phi}$. We can now use $f_{1}, f_{2}, g_{3}, \ldots, g_{r_{2}+1}$ to construct a map $f$ from the wedge $X$ of $r_{2}+1$ circles to Spec $R_{E}$ which has the properties claimed in proposition 2.1.

Now proposition 3.2 of [D-F1] tells us that $f$ induces a homotopy equivalence from $K\left(R_{E}\right)$ to a space $K(X)$, whose definition strictly depends not on $X$ as space but on the composite map

$$
X \stackrel{f}{\rightarrow} \operatorname{Spec} R_{E} \rightarrow \operatorname{Spec} \mathbb{Z}\left[\frac{1}{2}\right] .
$$

Again using the methods of [D-F1], we write $X=V_{1} \vee V_{2} \vee W$, where $V_{1}, V_{2}$ are the first two circles and $W$ is the wedge of the remaining ones. There is a fibre square

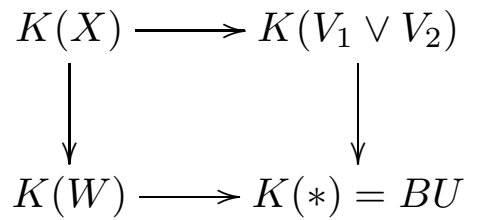

and $K(W)$, by the arguments of [D-F1] proposition 4.5, is the unpointed function space $B U^{W}=B U \times \prod^{r_{2}-1} U$. It follows easily that:

Proposition 2.2. The space $K(X)$ is homotopy equivalent to the product:

$$
K\left(V_{1} \vee V_{2}\right) \times \prod^{r_{2}-1} U
$$

And our main challenge is to identify the space $K\left(V_{1} \vee V_{2}\right)$. For this we have a second fibre square:

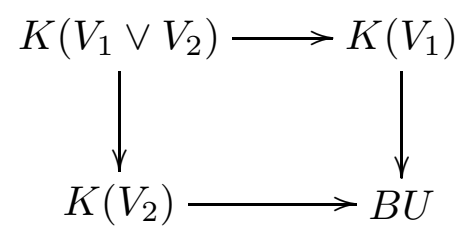

The space $K\left(V_{1}\right)$ is essentially well-known, and often used, being the 'finite fields' $K$-theory space $F \psi^{q}$ of Quillen [Q2]. It follows from the choice of the prime $\mathfrak{P}$ and the integer $q$ in $\S 1$ that the composite 
where the generator of $\pi_{1}\left(S^{1}\right)$ is mapped into the Frobenius of the finite field, can be taken for the inclusion of $V_{1}$ - its image under $\hat{\phi}$ is precisely $q$. We can therefore identify $K\left(V_{1}\right)$ with the 2-adic $K$-theory space of the finite field; and this is $F \psi^{q}$, the fibre of $\psi^{q}-1: B U \rightarrow B U$.

The following result is now not surprising, given its similarity to the foregoing:

Lemma 2.3. The space $K\left(V_{2}\right)$ is homotopy equivalent to the fibre $F \psi^{-1}$ of $\psi^{-1}-1$ : $B U \rightarrow B U$.

Proof. We give a rather ad hoc proof of what is probably a special case of a larger result. Let $R$ denote the 'ground ring' $\mathbb{Z}\left[\frac{1}{2}\right]$. The map $f_{2}: V_{2} \rightarrow \operatorname{Spec} R$, which defines $K\left(V_{2}\right)$ (see above), has an obvious double cover $\tilde{f}_{2}: \tilde{V}_{2} \rightarrow \operatorname{Spec} R$. However, $\tilde{f}_{2}$ is trivial on $\pi_{1}$, so that the corresponding space $K\left(\tilde{V}_{2}\right)$ is homotopy equivalent to $B U^{\tilde{V}_{2}}$ as before. ( $\tilde{V}_{2}$ is still a circle, of course.) Now the theory of [D-F2] tells us that $K\left(V_{2}\right)$ can be identified with the 'homotopy fixed points' $\operatorname{Hom}^{\Sigma}\left(E \Sigma, K\left(\tilde{V}_{2}\right)\right)$, where $\Sigma$ is the covering group $(=\mathbb{Z} / 2)$. In our case these are equivalent to the fixed points, i.e. to the equivariant maps $\lambda: \tilde{V}_{2} \rightarrow B U$; where $\Sigma$ acts on $\tilde{V}_{2}$ by the antipodal map, and on $B U$ by complex conjugation $\psi^{-1}$.

Clearly such a map $\lambda$ is determined by its values on the upper half-circle, and a standard argument shows that we can identify the space of such maps with the space of maps $\lambda^{\prime}:[0,1] \rightarrow B U$ satisfying $\lambda^{\prime}(1)=\psi^{-1}\left(\lambda^{\prime}(0)\right)$. But this is (one definition of) the homotopy fibre $F \psi^{-1}$.

Now we can compute $K\left(V_{1} \vee V_{2}\right)$. In fact, again following [D-F1], the wedge product gives rise to a fibre square, which we extend to the right:

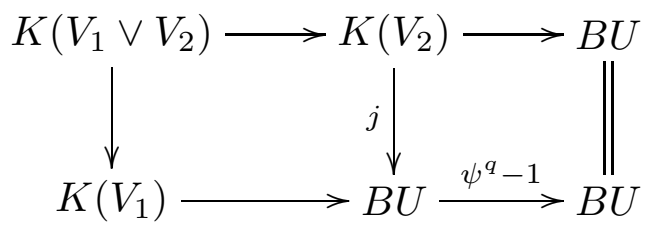

¿From this it is clear that $K\left(V_{1} \vee V_{2}\right)$ is the fibre of the composite $\left(\psi^{q}-1\right) \circ j$ : $F \psi^{-1} \rightarrow B U$, which is the space denoted $J_{c}(q)$ in $\S 1$. This identification, together with proposition 2.2, give us case (i) of theorem 1.1.

3. The case where $r_{1}>0$.

The case where $E$ admits real embeddings is (contrary to what one might expect) simplified by the fact that the ' $\mathbb{Z} / 2$ part' of the model space $X$ can be absorbed into the real embeddings and dealt with there. In this section we suppose $E$ 2-regular, with $r_{1}>0$; again we begin by producing a space $X$ which will serve as a model for the homology of Spec $R_{E}$. The homology (again adapted from [R- $\left.\varnothing\right]$ ) is described by:

Lemma 3.1. The mod 2 homology of $R_{E}$ is as follows:

$$
\begin{aligned}
& H_{1}\left(R_{E} ; \mathbb{Z} / 2\right)=\left(r_{1}+r_{2}+1\right) \cdot \mathbb{Z} / 2 \\
& H_{j}\left(R_{E} ; \mathbb{Z} / 2\right)=r_{1} \cdot \mathbb{Z} / 2 \quad(j>1)
\end{aligned}
$$

The $r_{1}$ real embeddings $R_{E} \stackrel{f_{i}}{\rightarrow} \mathbb{R}\left(i=1, \ldots, r_{1}\right)$ induce isomorphisms from the group 
The last sentence in the above statement is Tate's theorem: the higher homology of $R_{E}$ comes solely from the real embeddings. The fact that $E$ is 2 -regular makes it possible to extend the statement (true in general only for $j>2$ ) to the case $j=2$. As is the previous section, we can extend the statement to the $\mathbb{Z}_{2} \hat{\text { homology: }}$

Lemma 3.2. (i) The 2-completed homology of $R_{E}$ is as follows:

$$
\begin{aligned}
H_{1}\left(R_{E}\right) & =\left(r_{2}+1\right) \cdot \mathbb{Z}_{2} \oplus r_{1} \cdot \mathbb{Z} / 2 \\
H_{2 j}\left(R_{E}\right) & =0 \quad(j>0) \\
H_{2 j+1}\left(R_{E}\right) & =r_{1} \cdot \mathbb{Z} / 2 \quad(j>0)
\end{aligned}
$$

(ii) The $r_{1}$ real embeddings $R_{E} \stackrel{f_{i}}{\rightarrow} \mathbb{R}\left(i=1, \ldots, r_{1}\right)$ induce isomorphisms from the group $\oplus^{r_{1}} H_{j}(\mathbb{R})$ to $H_{j}\left(R_{E}\right)$ in dimensions $j>1$.

(iii) For $i=1, \ldots r_{1}$, the image of the non-trivial element of $H_{1}(\mathbb{R})$ under $\left(f_{i}\right)_{*}$ maps to $\sigma$ under $\hat{\phi}$.

Proof. Parts (i), (ii) require essentially the same as the proof of lemma 2.2. Part (iii) is a consequence of the statement that $\pi_{1}(\operatorname{Spec} \mathbb{R})=\mathbb{Z} / 2$ is generated by complex conjugation, which corresponds to $\sigma$.

Parallel to proposition 2.1 we have the following result setting up a space $X$ for this case.

Proposition 3.1. There is a map $f: X \rightarrow \operatorname{Spec} R_{E}$ such that:

(i) $X$ is a wedge of $r_{2}+1$ circles and $r_{1}$ copies of Spec $\mathbb{R}$;

(ii) The first circle, considered as an element of $\pi_{1}(X)$, maps to $q$ under the composite $\hat{\phi} \circ f_{*}$, and the rest (if any) map trivially;

(iii) For each copy of Spec $\mathbb{R}$, the non-trivial element of $\pi_{1}(\operatorname{Spec} \mathbb{R})$ maps to $\sigma \in$ $\left(\mathbb{Z}_{2} \hat{)}\right)^{*}$

(iv) $f$ induces an isomorphism on mod 2 homology.

Note. The copies of Spec $\mathbb{R}$ can be replaced by the infinite projective space $R P^{\infty} \sim$ $B \mathbb{Z} / 2$ (cf. [D-F1]), but this is not particularly useful for our purposes.

Proof. First note that the copies of $\operatorname{Spec} \mathbb{R}$ are already taken care of by lemma 3.2. Now as before consider the homomorphism from $H_{1}\left(R_{E}\right)$ onto $\Lambda \subset \operatorname{Aut}\left(\mu_{\infty}\right)$. Since the 2-torsion subgroup maps onto the subgroup generated by $\sigma \in \operatorname{Aut}\left(\mu_{\infty}\right)$, we can, by subtracting 2-torsion elements if necessary, choose a free direct summand $\left(r_{2}+1\right) \cdot \mathbb{Z}_{2} \hat{~ w h i c h ~ m a p s ~ e n t i r e l y ~ i n t o ~ t h e ~} \mathbb{Z}_{2} \hat{\text { part of }} \Lambda$. By choosing generators for this summand as in the proof of proposition 2.1, we can ensure that the first maps to $q$ and the others to zero.

Now represent these $\left(r_{2}+1\right)$ generators by maps $S^{1} \rightarrow \operatorname{Spec} R_{E}$. The wedge of these, and of our $r_{1}$ copies of Spec $\mathbb{R}$, with the given mappings into $\operatorname{Spec} R_{E}$, is the required space $X$.

We now have once again to find the space $K(X)$, representing wedges by fibred products over $B U$. However, we can speed up this process by dealing with all the copies of Spec $\mathbb{R}$ at once.

Proposition 3.2. Let $X_{1}, \ldots, X_{k}$ be copies of Spec $\mathbb{R}$ mapping into Spec $R_{E}$ by maps $f_{1}, \ldots, f_{k}$ which arise from real embeddings. Then $K\left(X_{1} \vee X_{2} \cdots \vee X_{k}\right)$ is 
from $K\left(X_{1} \vee X_{2} \cdots \vee X_{k}\right)$ to $B U$ is the complexification on $B O$, and the trivial map on each factor $U / O$.

Proof. First, by proposition 4.1 of [D-F1], we can identify $K(\mathbb{R})$ with $B O$, mapped into $B U$ in the usual way. We therefore need to find the fibred product of $k$ such copies of $B O$. To do this, replace the maps $B O \rightarrow B U$ by principal fibrations $p_{1}, \ldots, p_{k}$, with fibre $U / O$. A point of the fibred product is a sequence $\left(x_{1}, \ldots x_{k}\right)$ with $p_{1}\left(x_{1}\right)=\cdots=p_{k}\left(x_{k}\right)$. It follows that there are unique elements $g_{2}, \ldots g_{k}$ of $U / O$ such that $x_{i}=x_{1} \cdot g_{i}(i=2, \ldots, k)$; and that the map $\left(x_{1}, \ldots, x_{k}\right) \mapsto$ $\left(x_{1}, g_{2}, \ldots, g_{k}\right)$ is a homeomorphism from the fibred product to $B O \times \prod^{k-1}(U / O)$. The statement about the structure map is now obvious.

It is now relatively easy to complete the proof of theorem 1.1. Let us write $V$ for the wedge of the circles in the space $X$, and $W$ for the wedge of the Spec $\mathbb{R}$ 's. We know that the circles, as in the previous section (but here simplified by the absence of a $\mathbb{Z} / 2$-component) combine to give a space $K(V)=F \psi^{q} \times \prod^{r_{2}} U$; while $K(W)$ is given by the preceding result. To find the fibred product of $K(V)$ and $K(W)$ we need only look at those parts which are non-trivial over $B U$, i.e. at the $F \psi^{q}$ in $K(V)$ and the $B O$ in $K(W)$. Now the fibred product of these is again well-known, going back to Bökstedt and Dwyer-Friedlander; from the diagram

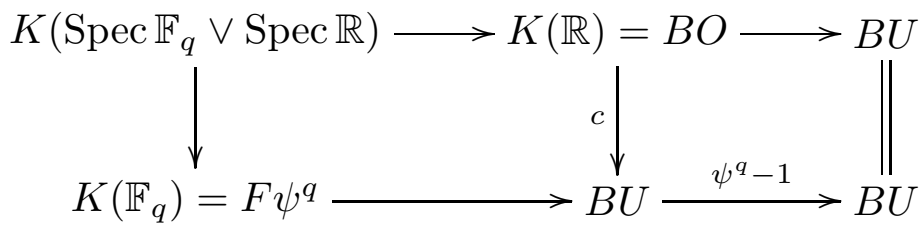

we see that it is precisely the fibre of the composite

$$
B O \stackrel{c}{\longrightarrow} B U \stackrel{\psi^{q}-1}{\longrightarrow} B U
$$

or what we have called $J K(q)$. Theorem 1.1 in the case of real embeddings follows immediately.

\section{The homotopy groups.}

As a check on the correctness of the preceding results, it makes sense to show that they imply the results on $K_{i}\left(R_{E}\right)=\pi_{i}\left(K\left(R_{E}\right)\right)$ proved in [R-Ø]. Some attention to details is needed since the homotopy groups of a fibre may not be uniquely determined by the exact sequence of the fibration (there may be extensions).

Again, we have to separate the two cases. In the totally imaginary case, since $\pi_{i}\left(\prod^{r_{2}-1} U\right)$ is well-known to be $\left(r_{2}-1\right) \cdot \mathbb{Z}_{2}$ for $i$ odd and 0 for $i$ even, the question reduces to finding the homotopy groups of the factor we have called $J_{c}(q)$, the homotopy fibre of the composite

$$
F \psi^{-1} \stackrel{j}{\rightarrow} B U \stackrel{\psi^{q}-1}{\longrightarrow} B U
$$

where $j$ is the fibre inclusion. The homotopy groups of $F \psi^{-1}$ (the classifying space of self-conjugate $K$-theory) were computed by D. Anderson in his thesis from 1963 , 
Lemma 4.1. The homotopy groups of $F \psi^{-1}$ are:

$$
\begin{aligned}
\pi_{4 k+1} & =\mathbb{Z} / 2 \\
\pi_{4 k+2} & =0 \\
\pi_{4 k+3} & =\mathbb{Z}_{2} \\
\pi_{4 k} & =\mathbb{Z}_{2}^{\hat{2}}
\end{aligned}
$$

and the fibre inclusion from $\mathrm{F}^{-1}$ to $\mathrm{BU}$ induces an isomorphism on $\pi_{4 k}$, and zero in all other cases.

Clearly we can deduce that $\left(\left(\psi^{q}-1\right) \circ j\right)_{*}$ is zero from $\pi_{i}\left(F \psi^{q}\right)$ to $\pi_{i}(B U)$ unless $i=4 k$, in which case it is multiplication by $q^{2 k}-1$ - this from the known action of the $\psi$ 's on the homotopy groups of $B U$.

It should be noted that $F \psi^{-1}$, or ' $K S C$ ' as it is traditionally called, is one of a family of $K$-spaces, and admits Adams operations $\psi^{q}$ for $q>1$ and odd, say. The fibre of $\psi^{q}-1: F \psi^{-1} \rightarrow F \psi^{-1}$ will be called $J S C(q)$, by an obvious analogy. Similarly, we use $J O(q)$ to denote the fibre of $\psi^{q}-1: B O \rightarrow B O$.

From the above, we can deduce:

Proposition 4.1. The homotopy groups of $J_{c}(q)$ are zero in even dimensions; for the odd ones we have:

$$
\pi_{4 k-1}\left(J_{c}(q)\right)=\mathbb{Z}_{2}^{\hat{2}} \oplus\left(\mathbb{Z} /\left(q^{2 k}-1\right)\right)_{2}
$$

and there is a split exact sequence

$$
0 \rightarrow \mathbb{Z}_{2}^{\hat{2}} \rightarrow \pi_{4 k+1}\left(J_{c}(q)\right) \rightarrow \mathbb{Z} / 2 \rightarrow 0
$$

Proof. The kernel of $\left(\left(\psi^{q}-1\right) \circ j\right)_{*}$ is $\mathbb{Z} / 2$ for $\pi_{4 k+1}, \mathbb{Z}_{2}$ for $\pi_{4 k-1}$, and zero otherwise, from the lemma; the cokernel is $\mathbb{Z}_{2}^{\hat{2}}$ for $\pi_{4 k+2},\left(\mathbb{Z} /\left(q^{2 k}-1\right)\right)_{2}$ for $\pi_{4 k}$, and zero otherwise. Hence it remains to prove that the exact sequence is split. Consider the digram of fibre sequences:

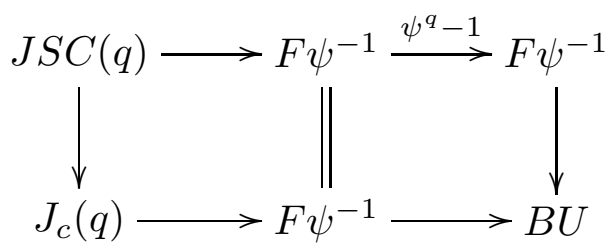

The map $\pi_{4 k+1}(J S C(q)) \rightarrow \pi_{4 k+1}\left(F \psi^{-1}\right)$ is an isomorphism by the choice of $q$, so we get the claimed splitting.

How does this fit with the results of $[\mathrm{R}-\varnothing]$ ? Once we have added $\left(r_{2}-1\right)$ copies of $\pi_{i}(U)$ as required, we have obvious agreement in the even dimensions. In dimension $4 k+1,[\mathrm{R}-\varnothing]$ give:

$$
\pi_{4 k+1}\left(K\left(R_{E}\right)\right)=r_{2} \cdot \mathbb{Z}_{2} \oplus \mathbb{Z} / w_{2 k+1}
$$

where $w_{2 k+1}=2$ is as defined in $\S 1$; hence our result agrees. In dimension $4 k+3$, their result is 
This agrees with our result provided we can identify $w_{2 k}$ with the 2-part of $q^{2 k}-1$. However, from the requirements on $q$ and on $w_{i}$ in $\S 1$, this is immediately evident.

We now consider the case where $r_{1}>0$. Here we need the homotopy groups of $U / O$; these are periodic of period 8 , as follows:

$$
\begin{aligned}
\pi_{8 k}(U / O) & =0 \\
\pi_{8 k+1}(U / O) & =\mathbb{Z}_{2} \\
\pi_{8 k+2}(U / O) & =\mathbb{Z} / 2 \\
\pi_{8 k+3}(U / O) & =\mathbb{Z} / 2 \\
\pi_{8 k+4}(U / O) & =0 \\
\pi_{8 k+5}(U / O) & =\mathbb{Z}_{2} \\
\pi_{8 k+6}(U / O) & =0 \\
\pi_{8 k+7}(U / O) & =0
\end{aligned}
$$

(These groups are well-known in the study of Bott periodicity; they also follow from identifying $U / O$ with $B\left(\mathbb{Z}_{2} \times B O\right)$.)

The homotopy groups $\pi_{i}\left(K\left(R_{E}\right)\right)=K_{i}\left(R_{E}\right)$ are the sum of $\left(r_{1}-1\right)$ copies of these, $r_{2}$ copies of $\pi_{i}(U)$, and $\pi_{i}(J K(q))$, which we must now determine. From the fibration

$$
J K(q) \rightarrow B O \stackrel{\left(\psi^{q}-1\right) \circ c}{\longrightarrow} B U
$$

we derive an exact homotopy sequence. All homomorphisms from $\pi_{i}(B O)$ to $\pi_{i}(B U)$ are necessarily zero (torsion to zero or free) except when $i=4 k$. It is known that $c_{*}$ is an isomorphism for $i=8 k$ and multiplication by 2 for $i=8 k+4$, and hence $\left(\left(\psi^{q}-1\right) \circ c\right)_{*}$ is multiplication by $q^{4 k}-1$ for $i=8 k$ and by $2\left(q^{4 k+2}-1\right)$ for $i=8 k+4$. From this (using the relation between $q$ and the $w_{i}$ 's as before) we deduce the following.

Proposition 4.2. The homotopy groups of $J K(q)$ are as follows:

$$
\begin{aligned}
\pi_{8 k} & =0 \\
\pi_{8 k+2} & =\mathbb{Z} / 2 \\
\pi_{8 k+3} & =\mathbb{Z} / 2 w_{4 k+2} \\
\pi_{8 k+4} & =0 \\
\pi_{8 k+5} & =\mathbb{Z} \hat{2} \\
\pi_{8 k+6} & =0 \\
\pi_{8 k+7} & =\mathbb{Z} / w_{4 k+4}
\end{aligned}
$$

and there is a split short exact sequence

$$
0 \rightarrow \mathbb{Z}_{2} \rightarrow \pi_{8 k+1} \rightarrow \mathbb{Z} / 2 \rightarrow 0
$$

Once again, these give exactly the results computed in $[R-\varnothing]$. 
(a) that the cokernel of $\left(\left(\psi^{q}-1\right) \circ c\right)_{*}$ is zero on odd dimensions, $\mathbb{Z}_{2}$ in dimension $4 k+2, \mathbb{Z} / 2 w_{4 k+2}$ in dimension $8 k+4$, and $\mathbb{Z} / w_{4 k+4}$ in dimension $8 k+8$;

(b) that the kernel is $\mathbb{Z} / 2$ in dimensions $8 k+1,8 k+2$, and zero otherwise.

Hence in the short exact sequence

$$
0 \rightarrow \operatorname{Coker}\left(\left(\psi^{q}-1\right) \circ c\right)_{*}(i+1) \rightarrow \pi_{i}(J K(q)) \rightarrow \operatorname{Ker}\left(\left(\psi^{q}-1\right) \circ c\right)_{*}(i) \rightarrow 0
$$

either the kernel or cokernel vanishes except for $i=8 k+1$. This therefore gives the only extension problem, and the other calculations are immediate. To solve the extension question we may look at the diagram of fibre sequences:

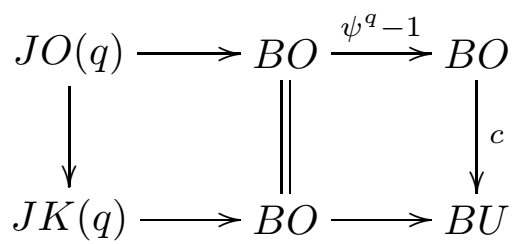

The map $\pi_{8 k+1}(J O(q)) \rightarrow \pi_{8 k+1}(B O)$ is a split surjection from $\mathbb{Z} / 2 \oplus \mathbb{Z} / 2$ to $\mathbb{Z} / 2$, and we are done.

\section{REFERENCES}

[At] M.F. Atiyah, K-theory, Benjamin, New York, 1967.

[B-L] S. Bloch and S. Lichtenbaum, A spectral sequence for motivic cohomology (preprint 1995).

[D-F1] W.G. Dwyer and E.M. Friedlander, Conjectural calculations of general linear group homology, Contemp. Math. vol. 55 part I, Amer.Math.Soc., Providence, 1986, pp. 135-147.

[D-F2] _ Algebraic and etale K-theory, Trans.Amer.Math.Soc. 292 (1985), 247-280.

[Mi1] S.A. Mitchell, Topological K-theory of algebraic K-theory spectra, K-Theory (2000) (to appear).

[Mi2] $\quad$ K-theory hypercohomology spectra of number rings at the prime 2, Arolla Conference Proceedings, 2000 (to appear).

[Q1] D.G. Quillen, Higher algebraic K-theory I, Lecture Notes in Math. 342, Springer, New York, 1973, pp. 85-147.

[Q2] On the cohomology and K-theory of the general linear group over finite fields, Annals of Math. 96 (1972), 552-586.

[R-W1] J. Rognes and C.A. Weibel, Two-primary algebraic K-theory of rings of integers in number fields, J. of Amer.Math.Soc. 13 (2000), 1-54.

[R-W2] _ Étale descent for two-primary algebraic K-theory of totally imaginary number rings, K-Theory 15 (1998), 101-104.

[R-Ø] J. Rognes and P.A. Østvær, K-theory of 2-regular number fields, Math.Z. 233 (2000), 251-263.

[S-V] A. Suslin and V. Voevodsky, Bloch-Kato conjecture and motivic cohomology with finite coefficients, The arithmetic and geometry of algebraic cycles, Proc. Conf., Banff 1998 (B.

B. Gordon, J. D. Lewis, S. Müller-Stach, S. Saito and N. Yui., eds.), Nato Science Series C, vol. 548, Kluwer, 2000, pp. 117-189.

[V] V. Voevodsky, The Milnor conjecture (preprint 1996).

[Ø1] P.A. Østvær, Étale descent for real number fields (part of thesis).

[Ø2] Algebraic K-theory spectra of non-exceptional two-regular number fields, Arolla Conference Proceedings, 2000 (to appear). 